\title{
Satellite Based Turbo-Coded, Blind-Equalized 4-QAM and 16-QAM Digital Video Broadcasting
}

\author{
Chee-Siong Lee, Spyros Vlahoyiannatos, and Lajos Hanzo, Senior Member, IEEE
}

\begin{abstract}
The Pan-European Digital Satellite Video Broadcasting (DVB-S) system's performance is characterized and improved with the aid of turbo coding and multi-level modulation. Specifically, various configurations of blind equalizers and convolutional as well as turbo codecs operating at different code rates were investigated. The standard system's performance was improved upon replacing the conventional convolutional codec by a turbo codec. Lastly, the feasibility of employing blind equalized 16-level modulation within the DVB-S system is demonstrated, potentially doubling the available bitrate and hence improving the associated video quality at the cost of a higher Signal-to-Noise Ratio (SNR) requirement. This extra transmitted power requirement can be eliminated upon invoking the more complex turbo codec, which requires lower transmitted power for attaining the same performance as the standard convolutional codes.
\end{abstract}

Index Terms-Blind equalization, digital video broadcasting, DVB, DVB-S, QAM, quadrature amplitude modulation.

\section{BACKGROUND AND MOTIVATION}

I N RECENT years three harmonized Digital Video Broadcasting (DVB) standards have emerged in Europe for terrestrial [1], cable-based [2], and satellite-oriented [3] delivery of DVB signals. The dispersive wireless propagation environment of the terrestrial system requires concatenated Reed-Solomon [4], [5] (RS) and rate compatible punctured convolutional coding [4], [5] (RCPCC) combined with Orthogonal Frequency Division Multiplexing (OFDM) based modulation [6]. The satellite-based system employs the same concatenated channel coding arrangement, as the terrestrial scheme, while the cable-based system refrains from using concatenated channel coding, opting for RS coding only. The performance of both of the latter schemes can be improved upon invoking blind-equalized multi-level modems [6], although the associated mild dispersion or linear distortion does not necessarily require channel equalization. However, since we propose invoking turbo-coded 4-bit/symbol 16-level quadrature amplitude modulation (16QAM) in order to improve the system's performance at the cost of increased complexity, in this contribution we additionally invoked blind channel equalizers. This is further justified by the associated high video transmission rates, where the dispersion may become a more dominant performance limitation.

Manuscript received November 22, 1999; revised February 15, 2000. This work was supported by EPSRC, U.K. in the framework of the Contract GR/K 74043 and the European Commission.

The authors are with the Department of Electronics and Computer Science, University of Southampton, SO17 1BJ, U.K. (e-mail: 1h@ecs.soton.ac.uk).

Publisher Item Identifier S 0018-9316(00)03932-9.
Lastly, the video codec used in all three systems is the Motion Pictures Expert Group's MPEG-2 codec. These standardization activities were followed by a variety of system performance studies in the open literature [7]-[10]. Against this background, in this paper we suggest turbo-coding based improvements to the satellite-based DVB system [3] and present performance studies of the proposed system under dispersive channel conditions in conjunction with a variety of blind channel equalization algorithms. The transmitted power requirements of the standard system employing convolutional codecs can be reduced upon invoking more complex, but more powerful turbo codecs. Alternatively, the standard quaternary or 2-bit/symbol system's bit error rate (BER) versus signal-to-noise ratio (SNR) performance can almost be matched by a turbo-coded 4-bit/symbol 16QAM based scheme, while doubling the achievable bit rate within the same bandwidth and hence improving the associated video quality. This is achieved at the cost of an increased system complexity.

The remainder of the paper is organized as follows. A succinct overview of the turbo-coded and standard DVB satellite scheme is presented in Section II, while our channel model is described in Section III. A brief summary of the blind equalizer algorithms employed is presented in Section IV. Following this the performance of the improved DVB satellite system is examined for transmission over a dispersive two-path channel in Section V, before our conclusions and future work areas are presented in Section VI.

\section{DVB SATElLite SCHEME}

The block diagram of the DVB satellite (DVB-S) system [3] is shown in Fig. 1, which is composed of an MPEG-2 video encoder (not shown in the diagram), channel coding modules and a quadrature phase shift keying (QPSK) modem [6]. The bitstream generated by the MPEG-2 encoder is packetized into frames of 188-bytes long. The video data in each packet is then randomized by the scrambler. The details concerning the scrambler have not been included in this paper, since these may be obtained from the DVB-S standard [3].

Due to the poor error resilience of the MPEG-2 video codec, powerful concatenated channel coding is employed. The concatenated channel codec comprises a shortened Reed-Solomon (RS) outer code and an inner convolutional encoder. The 188-byte MPEG-2 video packet is extended by the Reed-Solomon encoder [4], [5] with parity information to facilitate error recovery to form a 204-byte packet. The Reed-Solomon decoder can then correct up to eight erroneous bytes for each 204-byte packet. Following this, the RS-coded packet is interleaved by a convolutional interleaver and further 


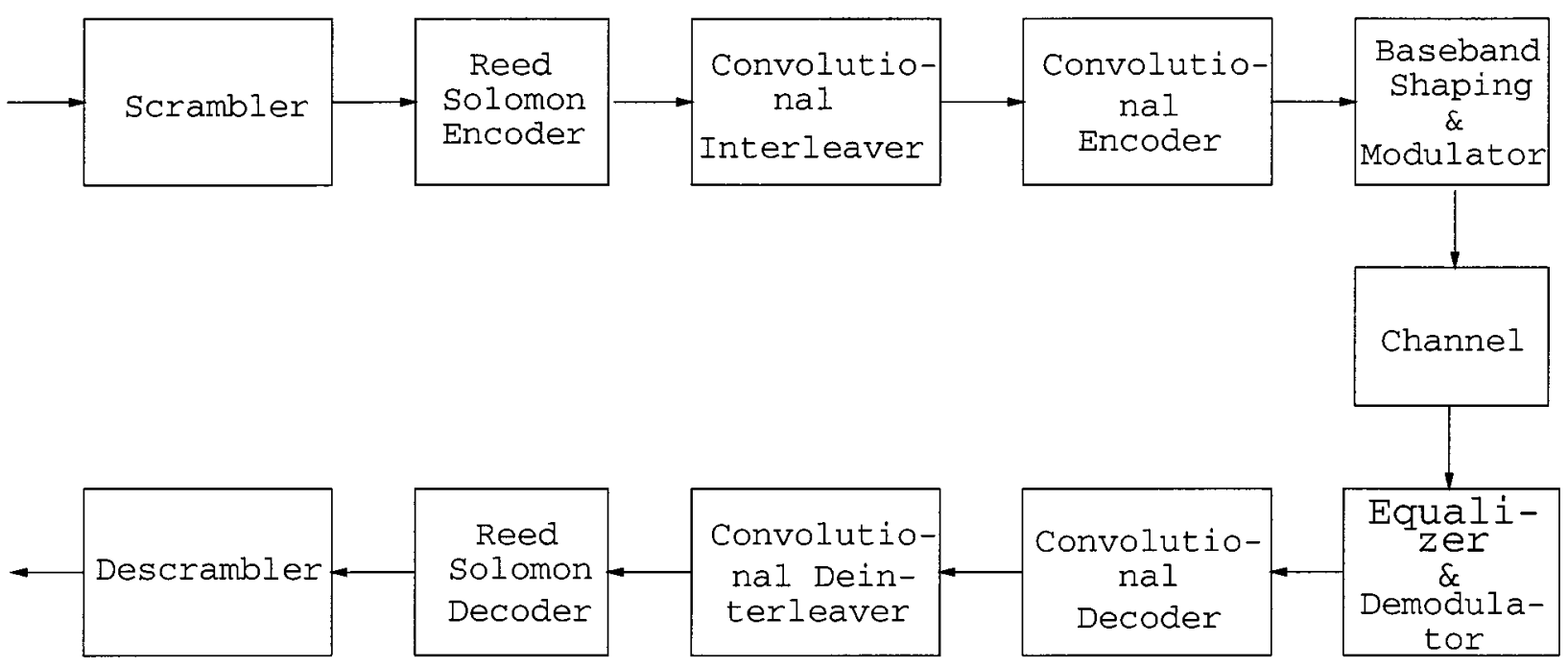

Fig. 1. Schematic of the DVB satellite system.

TABLE I

PARAMETERS OF THE $C C(n, k, K)$ CONVOLUTIONAL INNER ENCODER OF THE DVB-S MODEM

\begin{tabular}{|l|c|}
\hline Convolutional Coder Parameters \\
\hline Code Rate & $1 / 2$ \\
Constraint Length & 7 \\
$n$ & 2 \\
$k$ & 1 \\
Generator Polynomials (octal format) & 171,133 \\
\hline
\end{tabular}

TABLE II

PARAMETERS OF THE INNER TURBO ENCODER USED TO REPLACE THE DVB-S SYSTEM'S CONVOLUTIONAL CODER (RSC: RECURSIVE SYSTEMATIC CODE)

\begin{tabular}{|l|c|}
\hline \multicolumn{2}{|l|}{ Turbo Coder Parameters } \\
\hline Turbo Code Rate & $1 / 2$ \\
Input block length & 17952 bits \\
Interleaver Type & Random \\
Number of turbo decoder iterations & 8 \\
\hline \hline Turbo Encoder Component Code Parameters \\
\hline Component Code Encoder Type & Convolutional \\
& Encoder (RSC) \\
Component Code Decoder Type & Log-MAP[14] \\
Constraint Length & 3 \\
$n$ & 2 \\
$k$ & 1 \\
Generator Polynomials (octal format) & 7,5 \\
\hline
\end{tabular}

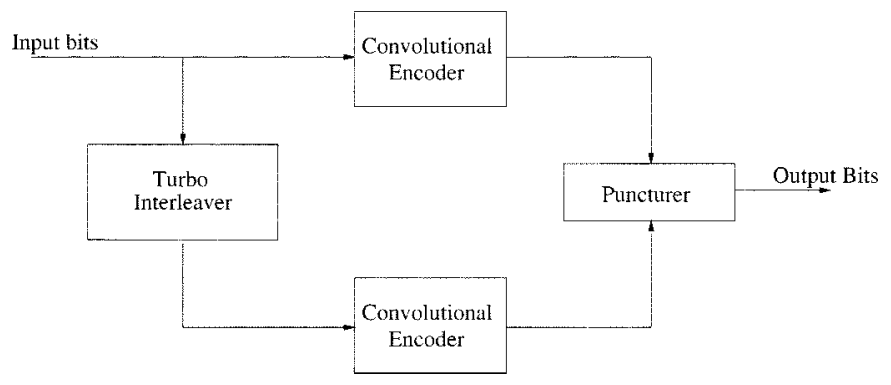

Fig. 2. Block diagram of turbo encoder. protected by a half-rate inner convolutional encoder with a constraint length of 7 [4], [5].

Furthermore, the overall code rate of the concatenated coding scheme can be adapted by variable puncturing, not shown in the figure, which supports code rates of $1 / 2$ (no puncturing) as well as $2 / 3,3 / 4,5 / 6$, and $7 / 8$. The parameters of the convolutional encoder are summarized in Table I.

In addition to implementing the standard DVB-S system as a benchmark, we have improved the system's performance with the aid of a turbo codec [11], [12]. The block diagram of the turbo encoder is shown in Fig. 2. The turbo encoder is constructed of two component encoders. Each component encoder is a half-rate convolutional encoder, whose parameters are listed in Table II. The two component encoders are used to encode the same input bits, although the input bits of the second component encoder are interleaved before encoding. The output bits of the two component codes are punctured and multiplexed, in order to form a single output bitstream. The component encoder used here is known as a half-rate recursive systematic convolutional encoder (RSC) [13]. It generates one parity bit and one systematic output bit for every input bit. In order to provide an overall coding rate of one half, half the output bits from the two encoders must be punctured. The puncturing arrangement used in our work is to transmit all the systematic bits from the first encoder and every other parity bit from both encoders.

Readers interested in further details of the DVB-S system are referred to the DVB-S standard [3]. The performance of the standard DVB-S system and that of the turbo coded system is characterized in Section V. Let us now briefly consider the multipath channel model used in our investigations.

\section{Channel Model}

The DVB-S system was designed to operate in the $12 \mathrm{GHz}$ frequency band (K-band). Within this frequency band, tropospheric effects such as the transformation of electromagnetic energy into thermal energy due to induction of currents in rain and ice crystals lead to signal attenuations [15], [16]. In the 
TABLE III

SuMmary OF THE EQUalizer PaRAMETERS USED IN THE SimUlations. THE TAP-VeCtOR $(1.2,0, \cdots, 0)$ INDICATES THAT THE FIRST EQUALIZER COEFFICIENT Is INITIALIZED to THE VALUE 1.2 , While THE OTHERS to 0

\begin{tabular}{|c|c|c|c|}
\hline & $\begin{array}{c}\text { Step-size } \\
\lambda\end{array}$ & $\begin{array}{c}\text { No. of } \\
\text { Equal. } \\
\text { Taps }\end{array}$ & $\begin{array}{c}\text { Initial } \\
\text { Tap- } \\
\text { Vector }\end{array}$ \\
\hline Benveniste-Goursat & $5 \times 10^{-4}$ & 10 & $(1.2,0, \cdots, 0)$ \\
\hline Modified-CMA & $5 \times 10^{-4}$ & 10 & $(1.2,0, \cdots, 0)$ \\
\hline Stop-and-Go & $5 \times 10^{-4}$ & 10 & $(1.2,0, \cdots, 0)$ \\
\hline PSP (1 sym delay) & $10^{-2}$ & 2 & $(1.2,0)$ \\
\hline PSP (2 sym delay) & $10^{-2}$ & 3 & $(1.2,0,0)$ \\
\hline
\end{tabular}

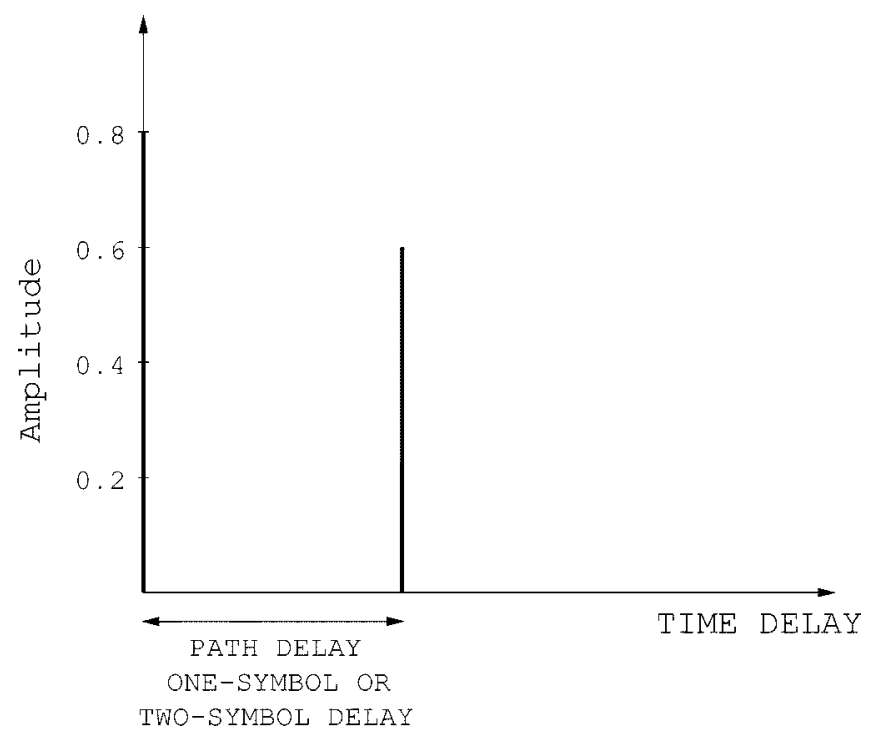

Fig. 3. Two-path satellite channel model with either a one-symbol or two-symbol delay.

past 20 years, various researchers have concentrated their efforts on attempting to model the satellite channel, typically within a land mobile satellite channel scenario. However, the majority of the work conducted for example by Vogel and his colleagues [17]-[20], concentrated on modeling the statistical properties of a narrowband satellite channel in lower frequency bands, such as the $870 \mathrm{MHz}$ UHF band and the $1.5 \mathrm{GHz}$ L-band.

However, our high bitrate DVB satellite system requires a high bandwidth, hence the video bitstream is exposed to dispersive wideband propagation conditions. Recently, Saunders et al. [21], [22] have proposed the employment of multipath channel models to study the satellite channel, although their study was concentrated on the L-band and S-band only.

Due to the dearth of reported work on wideband satellite channel modeling in the K-band, we have adopted a simpler approach. The channel model employed in this study was the two-path $(n T)$-symbol spaced impulse response, where $T$ is the symbol-duration. In our studies we used $n=1$ and $n=2$. This corresponds to a stationary dispersive transmission channel. Our channel model assumed that the receiver had a direct line-ofsight with the satellite as well as a second path caused by a single reflector probably from a nearby building or due to ground reflection. The ground reflection may be strong, if the satellite receiver dish is only tilted at a low angle.

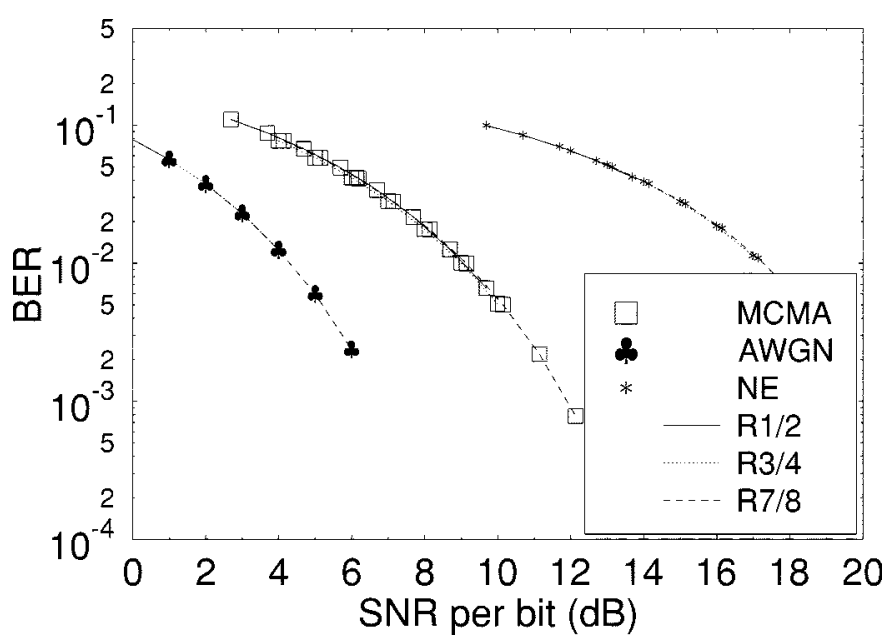

(a)

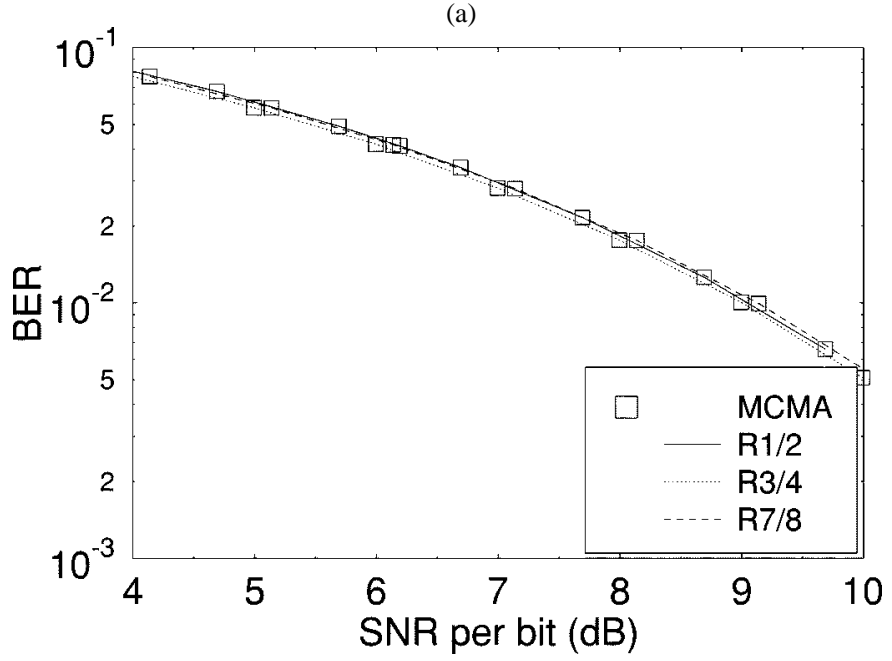

(b)

Fig. 4. Average BER versus SNR per bit performance after equalization and demodulation employing QPSK modulation and one-symbol delay channel (NE: Non-Equalized; MCMA: Modified Constant Modulus Algorithm). (a) After equalization and demodulation; (b) Same as (a) but enlarged in order to show performance difference of the blind equalizer, when different convolutional code rates are used.

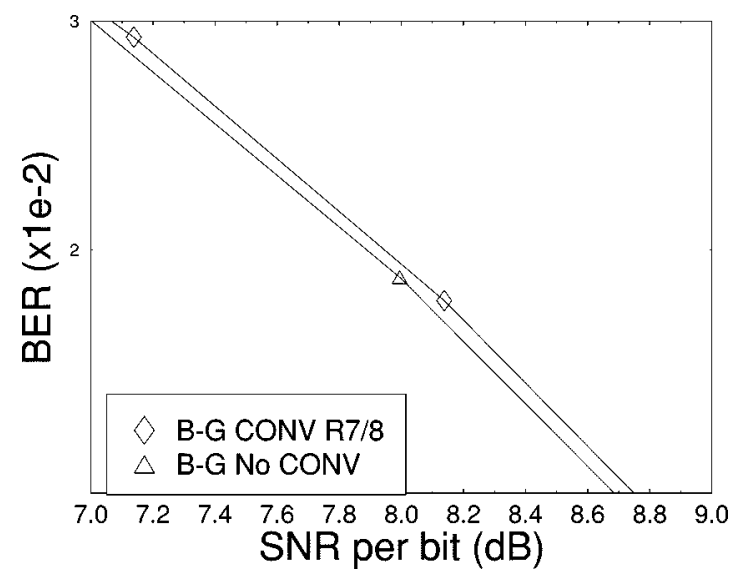

Fig. 5. Average BER versus SNR per bit performance after equalization and demodulation employing QPSK modulation and the one-symbol delay two-path channel of Fig. 3, for the Benveniste-Goursat algorithm, where the input bits are random (No CONV) or correlated (CONV 7/8) as a result of convolutional coding having a coding rate of $7 / 8$. 

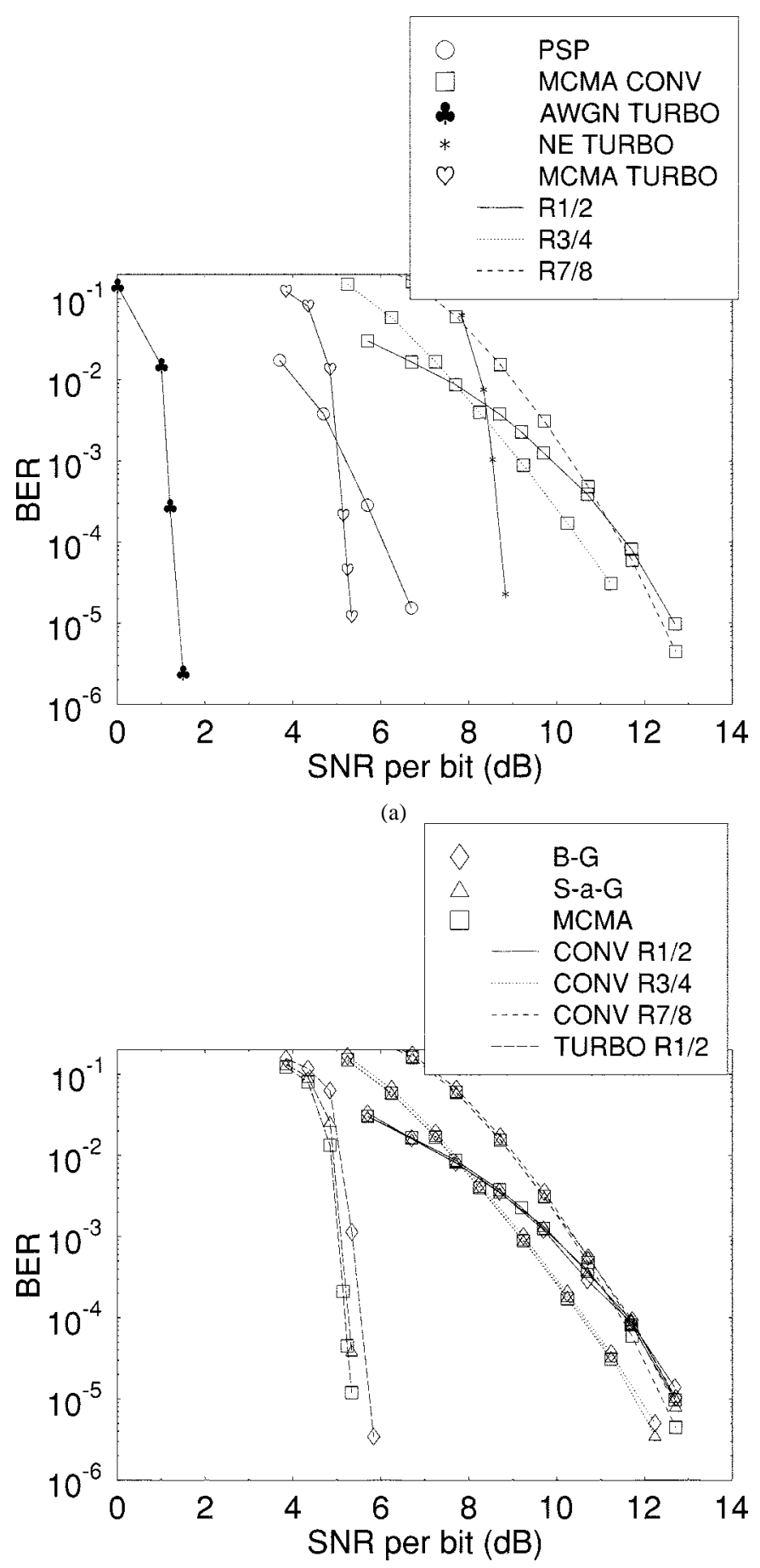

(b)

Fig. 6. Average BER versus SNR per bit performance after convolutional or turbo decoding for QPSK modulation and one-symbol delay channel (NE: Non-Equalized; B-G: Benveniste-Goursat; S-a-G: Stop-and-Go; MCMA: Modified Constant Modulus Algorithm; PSP: Per-Survivor-Processing). (a) PSP and linear equalizers; (b) Linear equalizers only.

Based on these channel models, we studied the ability of a range of blind equalizer algorithms to converge under various path delay conditions. In the next section we provide a brief overview of the various blind equalizers employed in our experiments, noting that the readers who are mainly interested in the system's performance may proceed directly to our performance analysis section, namely to Section V.

\section{THE BLIND EQUALIZERS}

In this section the blind equalizers used in the system are presented. The following blind equalizers have been studied:

- The Modified Constant Modulus Algorithm (MCMA) [23]

- The Benveniste-Goursat Algorithm (B-G) [24]

- The Stop-and-Go Algorithm (S-a-G)[25]

- The Per-Survivor Processing (PSP) Algorithm [26].

We will now briefly introduce these algorithms. First we define the variables that we will use:

$$
\begin{gathered}
\boldsymbol{y}(n)=\left[y\left(n+N_{1}\right), \cdots, y(0), \cdots, y\left(n-N_{2}\right)\right]^{T} \\
\boldsymbol{c}^{(n)}=\left[c_{-N_{1}}, \cdots, c_{o}, \cdots, c_{N_{2}}\right]^{T} \\
z(n)=\left(\boldsymbol{c}^{(n)}\right)^{T} \boldsymbol{y}(n)=\boldsymbol{y}^{T}(n) \cdot \boldsymbol{c}^{(n)}
\end{gathered}
$$

where $\boldsymbol{y}(n)$ is the received symbol vector at time $n$, containing the $N_{1}+N_{2}+1$ most recent received symbols, while $N_{1}, N_{2}$ are the number of equalizer feedback and feedforward taps, respectively. Furthermore, $c^{(n)}$ is the equalizer tap-vector, consisting of the equalizer tap values and $z(n)$ is the equalized symbol at time $n$, given by the convolution of the received signal with the equalizers impulse response, while ()$^{T}$ stands for matrix transpose. Note that the variables of (1)-(3) assume complex values, when multi-level modulation is employed.

The Modified CMA (MCMA) is an improved version of Godard's well-known Constant Modulus Algorithm (CMA) [27]. The philosophy of the CMA is based on forcing the magnitude of the equalized signal to a constant value. In mathematical terms the CMA is based on minimizing the cost function:

$$
J^{(\mathrm{CMA})}=E\left[\left(|z(n)|^{2}-R_{2}\right)^{2}\right]
$$

where $R_{2}$ is a suitably chosen constant and $E$ [] stands for the expectation. Similarly to the CMA, the MCMA, which was proposed by Wesolowsky [23], forces the real and imaginary parts of the complex signal to the constant values of $R_{2, R}$ and $R_{2, I}$, respectively, according to the equalizer tap update equation of [23]:

$$
\begin{aligned}
\boldsymbol{c}^{(n+1)}= & \boldsymbol{c}^{(n)}-\lambda \cdot \boldsymbol{y}^{*}(n) \\
& \cdot\left\{\operatorname{Re}[z(n)]\left((\operatorname{Re}[z(n)])^{2}-R_{2, R}\right)\right. \\
& \left.+j \cdot \operatorname{Im}[z(n)]\left((\operatorname{Im}[z(n)])^{2}-R_{2, I}\right)\right\},
\end{aligned}
$$

where $\lambda$ is the step-size parameter and the $R_{2, R}, R_{2, I}$ constant parameters of the algorithm are defined as:

$$
\begin{aligned}
& R_{2, R}=\frac{E\left[(\operatorname{Re}[a(n)])^{4}\right]}{E\left[(\operatorname{Re}[a(n)])^{2}\right]} \\
& R_{2, I}=\frac{E\left[(\operatorname{Im}[a(n)])^{4}\right]}{E\left[(\operatorname{Im}[a(n)])^{2}\right]},
\end{aligned}
$$



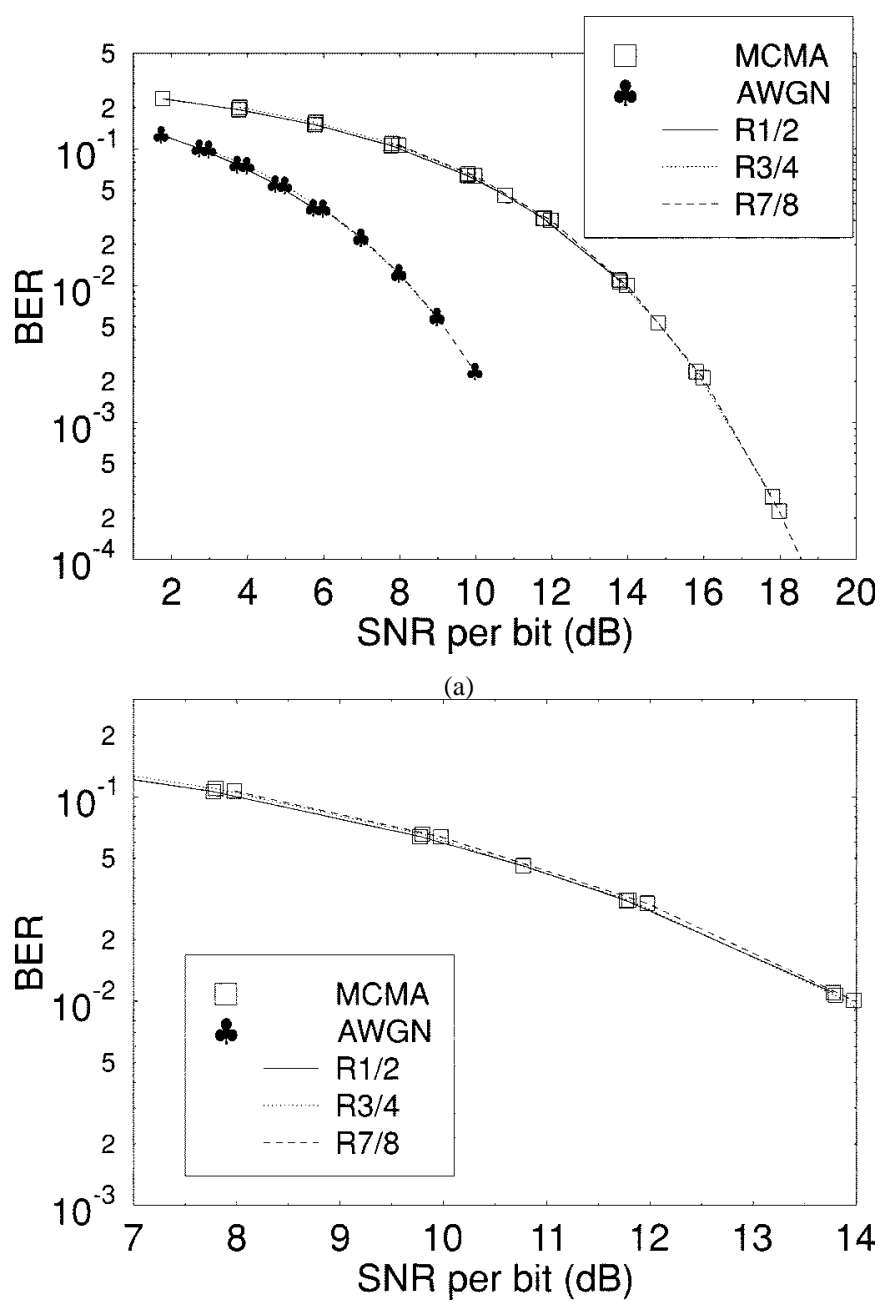

(b)

Fig. 7. Average BER versus SNR per bit after equalization and demodulation for 16-QAM over the one-symbol delay two-path channel of Fig. 3 (MCMA: Modified Constant Modulus Algorithm). (a) After equalization and demodulation; (b) Same as (a) but enlarged in order to show performance difference of the blind equalizer, when different convolutional code rates are used.

where $a(n)$ is the transmitted signal at time instant $n$.

The Benveniste-Goursat (B-G) algorithm [24] is an amalgam of the Sato's algorithm [28] and the decision-directed (DD) algorithm [6]. Strictly speaking, the decision-directed algorithm is not a blind equalization technique, since its convergence is highly dependent on the channel.

This algorithm estimates the error between the equalized signal and the detected signal as:

$$
\epsilon^{\mathrm{DD}}(n)=z(n)-\hat{z}(n)
$$

where $\hat{z}(n)$ is the receiver's estimate of the transmitted signal at time instant $n$. Similarly to the DD algorithm's error term, the Sato-type error [28] is defined as:

$$
\epsilon^{\mathrm{Sato}}(n)=z(n)-\gamma \cdot \operatorname{csgn}(z(n))
$$

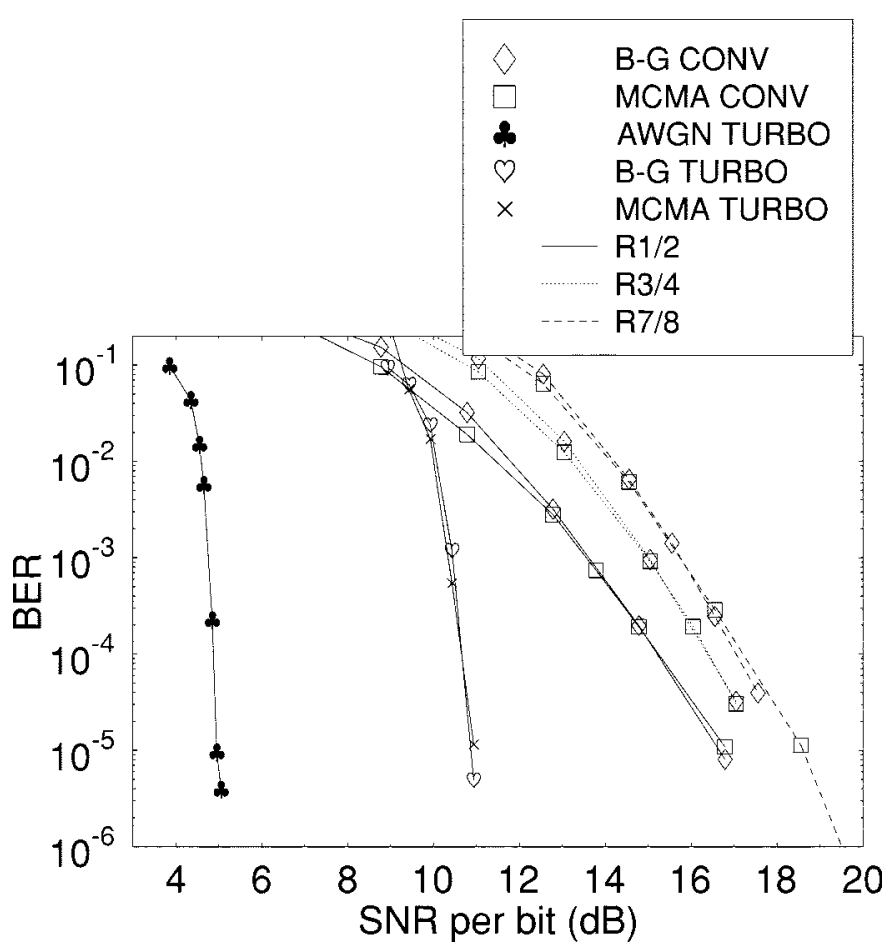

Fig. 8. Average BER versus SNR per bit after Viterbi or turbo decoding for 16-QAM over the one-symbol delay two-path channel of Fig. 3 (B-G: Benveniste-Goursat; S-a-G: Stop-and-Go; MCMA: Modified Constant Modulus Algorithm; PSP: Per-Survivor-Processing).

where $\gamma$ is a constant parameter of the Sato-algorithm, defined as:

$$
\gamma=\frac{E\left[(\operatorname{Re}[a(n)])^{2}\right]}{E[|\operatorname{Re}[a(n)]|]}=\frac{E\left[(\operatorname{Im}[a(n)])^{2}\right]}{E[|\operatorname{Im}[a(n)]|]}
$$

and $\operatorname{csgn}(x)=\operatorname{sign}(\operatorname{Re}\{x\})+j \cdot \operatorname{sign}(\operatorname{Im}\{x\})$ is the complex sign function. The $\mathrm{B}-\mathrm{G}$ algorithm combines the above two error terms into one:

$$
\epsilon^{G}(n)=k_{1} \cdot \epsilon^{\mathrm{DD}}(n)+k_{2} \cdot\left|\epsilon^{\mathrm{DD}}(n)\right| \cdot \epsilon^{\mathrm{Sato}}(n),
$$

where the two error terms are suitably weighted by the constant parameters $k_{1}$ and $k_{2}$ in (11). Using this error term, the $\mathrm{B}-\mathrm{G}$ equalizer updates the equalizer coefficients according to the following equalizer tap update equations [24]:

$$
\boldsymbol{c}^{(n+1)}=\boldsymbol{c}^{(n)}-\lambda \cdot \boldsymbol{y}^{*}(n) \cdot \epsilon^{G}(n) .
$$

In our investigations, the weights were chosen as $k_{1}=1, k_{2}=$ 5 , so that the Sato-error was weighted more heavily, than the DD-error.

The Stop-and-Go (S-a-G) algorithm [25] is a variant of the decision-directed algorithm [6], where at each equalizer coefficient adjustment iteration the update is enabled or disabled, depending on whether the update is likely to be correct. The update equations of this algorithm are given by [25]:

$$
\begin{aligned}
\boldsymbol{c}^{(n+1)}=\boldsymbol{c}^{(n)}-\lambda \cdot \boldsymbol{y}^{*}(n)[ & f_{n, R} \operatorname{Re}\left\{\epsilon^{D D}(n)\right\} \\
& \left.+j f_{n, I} \operatorname{Im}\left\{\epsilon^{D D}(n)\right\}\right]
\end{aligned}
$$

where * stands for the complex conjugate, $\epsilon^{\mathrm{DD}}(n)$ is the decision-directed error as in (8) and the binary functions $f_{n, R}$, 


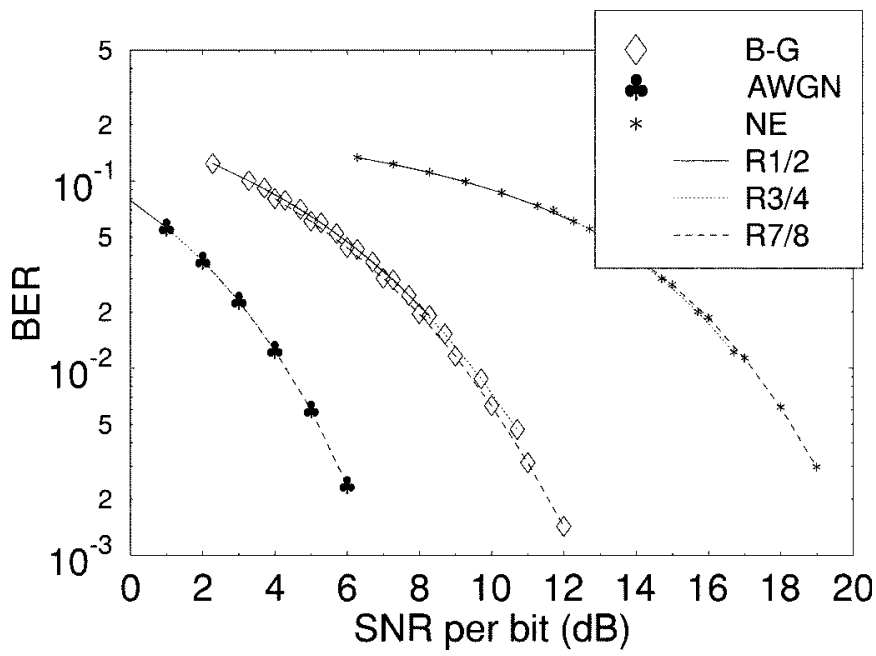

(a)

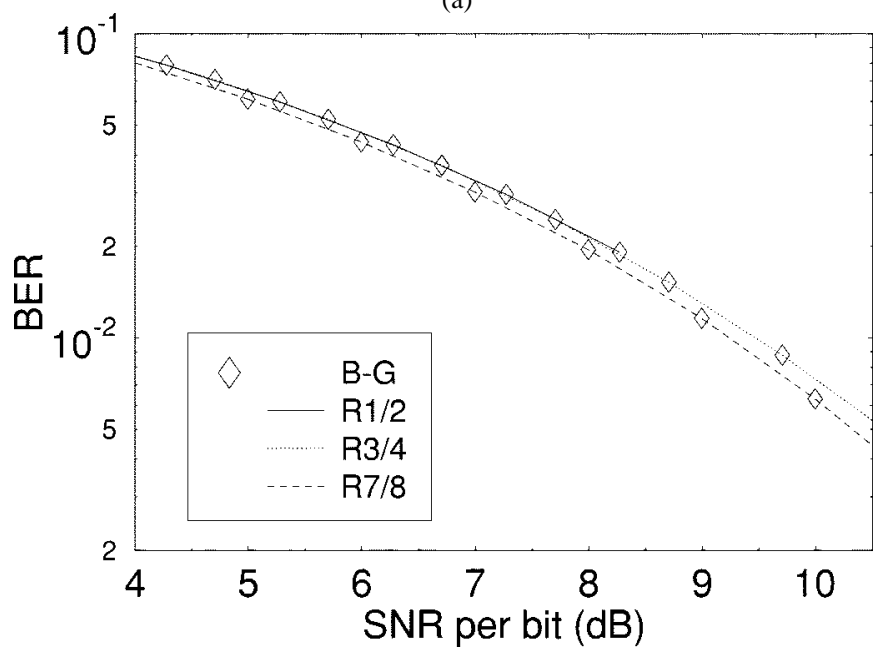

(b)

Fig. 9. Average BER versus SNR per bit performance after equalization and demodulation for QPSK modulation over the two-symbol of Fig. 3 (B-G: Benveniste-Goursat). (a) After equalization and demodulation; (b) Same as (a) but enlarged in order to show performance difference of the blind equalizer, when different convolutional code rates are used.

$f_{n, I}$ enable or disable the update of the equalizer according to the following rule. If the sign of the Sato-error (the real or the imaginary part independently) is the same as the sign of the decision-directed error, then the update takes place, otherwise it does not.

In mathematical terms, this is equivalent to [25]:

$$
f_{n, R}= \begin{cases}1 & \text { if } \operatorname{sgn}\left(\operatorname{Re}\left[\epsilon^{\mathrm{DD}}(n)\right]\right)=\operatorname{sgn}\left(\operatorname{Re}\left[\epsilon^{\text {Sato }}(n)\right]\right) \\ 0 & \text { if } \operatorname{sgn}\left(\operatorname{Re}\left[\epsilon^{\mathrm{DD}}(n)\right] \neq \operatorname{sgn}\left(\operatorname{Re}\left[\epsilon^{\text {Sato }}(n)\right]\right)\right.\end{cases}
$$

$$
f_{n, I}= \begin{cases}1 & \text { if } \operatorname{sgn}\left(\operatorname{Im}\left\{\epsilon^{\mathrm{DD}}(n)\right\}\right)=\operatorname{sgn}\left(\operatorname{Im}\left\{\epsilon^{\text {Sato }}(n)\right\}\right) \\ 0 & \text { if } \operatorname{sgn}\left(\operatorname{Im}\left\{\epsilon^{\mathrm{DD}}(n)\right\}\right) \neq \operatorname{sgn}\left(\operatorname{Im}\left\{\epsilon^{\text {Sato }}(n)\right\}\right) .\end{cases}
$$

For a blind equalizer, this condition provides us with a measure of the probability of the coefficient update being correct.

The PSP algorithm [26] is based on employing convolutional coding and hence it is a trellis-based sequence estimation technique, in which the channel is not known "a priori." Hence, an iterative channel estimation technique is employed, in order to estimate the channel jointly with the modulation symbol. In this sense, an initial channel estimation is used and the estimation is updated at each new symbol's arrival.

In our case the update was based on the Least Mean Squares $(L M S)$ estimates, according to the following channel-tap update equations [26]:

$$
\hat{\boldsymbol{h}}^{(n+1)}=\hat{\boldsymbol{h}}^{(n)}+\lambda \cdot \hat{\boldsymbol{a}}^{*}(n) \cdot\left(y(n)-\hat{\boldsymbol{a}}^{T}(n) \cdot \hat{\boldsymbol{h}}^{(n)}\right),
$$

where

$$
\hat{\boldsymbol{h}}^{(n)}=\left(\hat{h}_{-L_{1}}^{(n)}, \cdots, \hat{h}_{o}^{(n)}, \cdots, \hat{h}_{L_{2}}^{(n)}\right)^{T}
$$

is the estimated (for one surviving path) channel tap-vector at time instant $n$,

$$
\hat{\boldsymbol{a}}(n)=\left(\hat{a}\left(n+L_{1}\right), \cdots, \hat{a}(0), \cdots, \hat{a}\left(n-L_{2}\right)\right)^{T}
$$

is the associated estimated transmitted symbol vector and $y(n)$ is the actually received symbol at time instant $n$.

Each of the surviving paths in the trellis carries not only its own signal estimation, but also its own channel estimation. Moreover, convolutional decoding can take place jointly with this channel and data estimation procedure, leading to improved Bit Error Rate (BER) performance. A summary of the various equalizers' parameters is given in Table III.

Having described the components of our enhanced DVB-S system, let us now consider the overall system's performance.

\section{Performance of the DVB-S Scheme}

In this section, the performance of the DVB-S system was evaluated by means of simulations. Two modulation types were used, namely the standard QPSK and the enhanced 16-QAM schemes [6]. The channel model of Fig. 3 was employed. The first channel model had a one-symbol second-path delay, while in the second one the path-delay corresponded to the period of two symbols. The average BER versus SNR per bit performance was evaluated after the equalization and demodulation process, as well as after Viterbi [4] or turbo decoding [12]. The SNR per bit or $E_{b} / N_{o}$ is defined as follows:

$$
\text { SNR per bit }=10 \log _{10} \frac{\bar{S}}{\bar{N}}+\delta
$$

where $\bar{S}$ is the average received signal power, $\bar{N}$ is the average received noise power and $\delta$, which is dependent on the type of modulation scheme used and channel code rate $(\mathrm{R})$, is defined as follows:

$$
\delta=10 \log _{10} \frac{1}{\mathrm{R} \times \text { Bits per modulation symbol }} .
$$

Our results are further divided into two subsections for ease of discussion. First, we will present the system performance over the one-symbol delay two-path channel in Section V-A. Next, the system performance over the two-symbol delay two-path channel is presented in Section V-B. Lastly, a summary of the system performance is provided in Section V-C. 

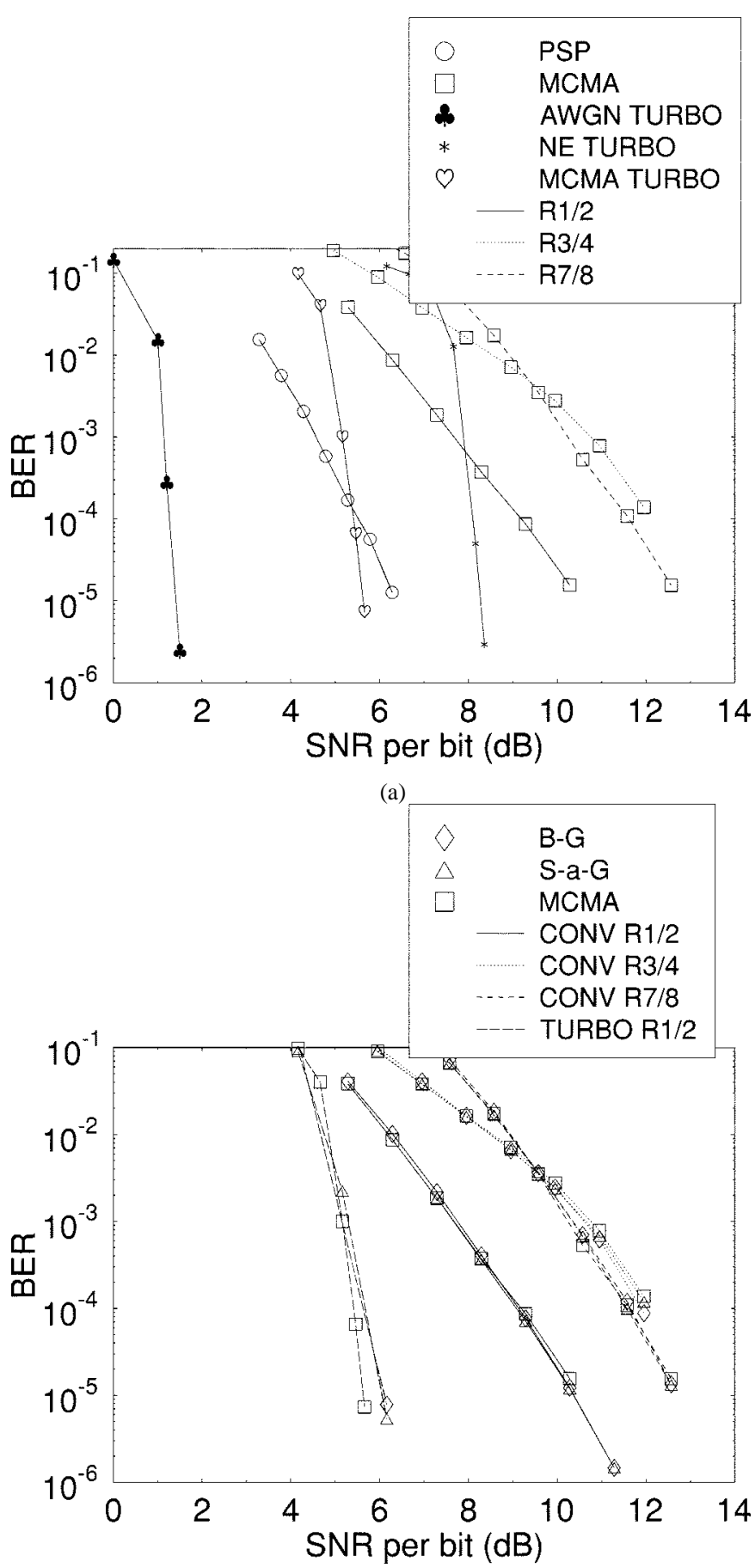

(b)

Fig. 10. Average BER versus SNR per bit performance after convolutional or turbo decoding for QPSK modulation over the two-symbol delay two-path channel of Fig. 3 (B-G: Benveniste-Goursat; S-a-G: Stop-and-Go; MCMA: Modified Constant Modulus Algorithm; PSP: Per-Survivor-Processing). (a) PSP and linear equalizers; (b) Linear equalizers only.

\section{A. Transmission Over the Symbol-Spaced Two-Path Channel}

The linear equalizers' performance was quantified and compared using QPSK modulation over the one-symbol delay two-path channel model of Fig. 4. Since all the equalizers' BER performance was similar, only the Modified CMA results are shown in the figure.
The equalized performance over the one symbol-spaced channel was inferior to that over the nondispersive AWGN channel. However, as expected, it was better than without any equalization. Another observation for Fig. 4 was that the different punctured channel coding rates appeared to give slightly different bit error rates after equalization. This was because the linear blind equalizers required uncorrelated input bits in order to converge. However, the input bits were not entirely random, when convolutional coding was used. The consequences of violating the zero-correlation constraint are not generally known. Nevertheless, two potential problems were apparent. First, the equalizer may diverge from the desired equalizer equilibrium [29]. Secondly, the performance of the equalizer is expected to degrade, owing to the violation of the randomness requirement, which is imposed on the input bits in order to ensure that the blind equalizers will converge.

Since the channel used in our investigations was static, the first problem was not encountered. Instead, the second problem was what we actually observed. Fig. 5 quantifies the equalizers' performance degradation due to convolutional coding. We can observe a $0.1 \mathrm{~dB}$ SNR degradation, when the convolutional codec creates correlation among the bits for this specific case.

The average BER curves after Viterbi or turbo decoding are shown in Fig. 6(a). In this figure, the average BER over the nondispersive AWGN channel after turbo decoding constitutes the best case performance, while the average BER of the onesymbol delay two-path MCMA-equalized rate 7/8 convolutionally coded scenario exhibits the worst case performance. Again, in this figure only the Modified-CMA was featured for simplicity. The performance of the remaining equalizers was characterized in Fig. 6(b). Clearly, the performance of all the linear equalizers investigated was similar.

It is observed in Fig. 6(a) that the combination of the Modified CMA blind equalizer with turbo decoding exhibited the best SNR performance over the one-symbol delay two-path channel. The only comparable alternative was the PSP algorithm. Although the performance of the PSP algorithm was better at low SNR's, the associated curves cross over and the PSP algorithm's performance became inferior below the average BER of $10^{-3}$. Although not shown in Fig. 6, the Reed-Solomon decoder, which was concatenated to either the convolutional or the turbo decoder, became effective, when the average BER of its input was below approximately $10^{-4}$. In this case, the PSP algorithm performed by at least $1 \mathrm{~dB}$ worse in the area of interest, which is at an average BER of $10^{-4}$.

A final observation in the context of Fig. 6(a) is that when convolutional decoding was used, the associated $E_{b} / N_{o}$ performance of the rate $1 / 2$ convolutional coded scheme appeared slightly inferior to that of the rate $3 / 4$ and the rate $7 / 8$ scenarios beyond certain $E_{b} / N_{\circ}$ values. This was deemed to be a consequence of the fact that the 1/2-rate encoder introduced more correlation into the bitstream than its higher rate counterparts and this degraded the performance of the blind channel equalizers, which performed best, when fed with random bits.

Having considered the QPSK case, we shall now concentrate on the enhanced system, which employed 16-QAM under the same channel and equalizer conditions. In Figs. 7 and 8, the performance of the DVB system employing 16-QAM is presented. 

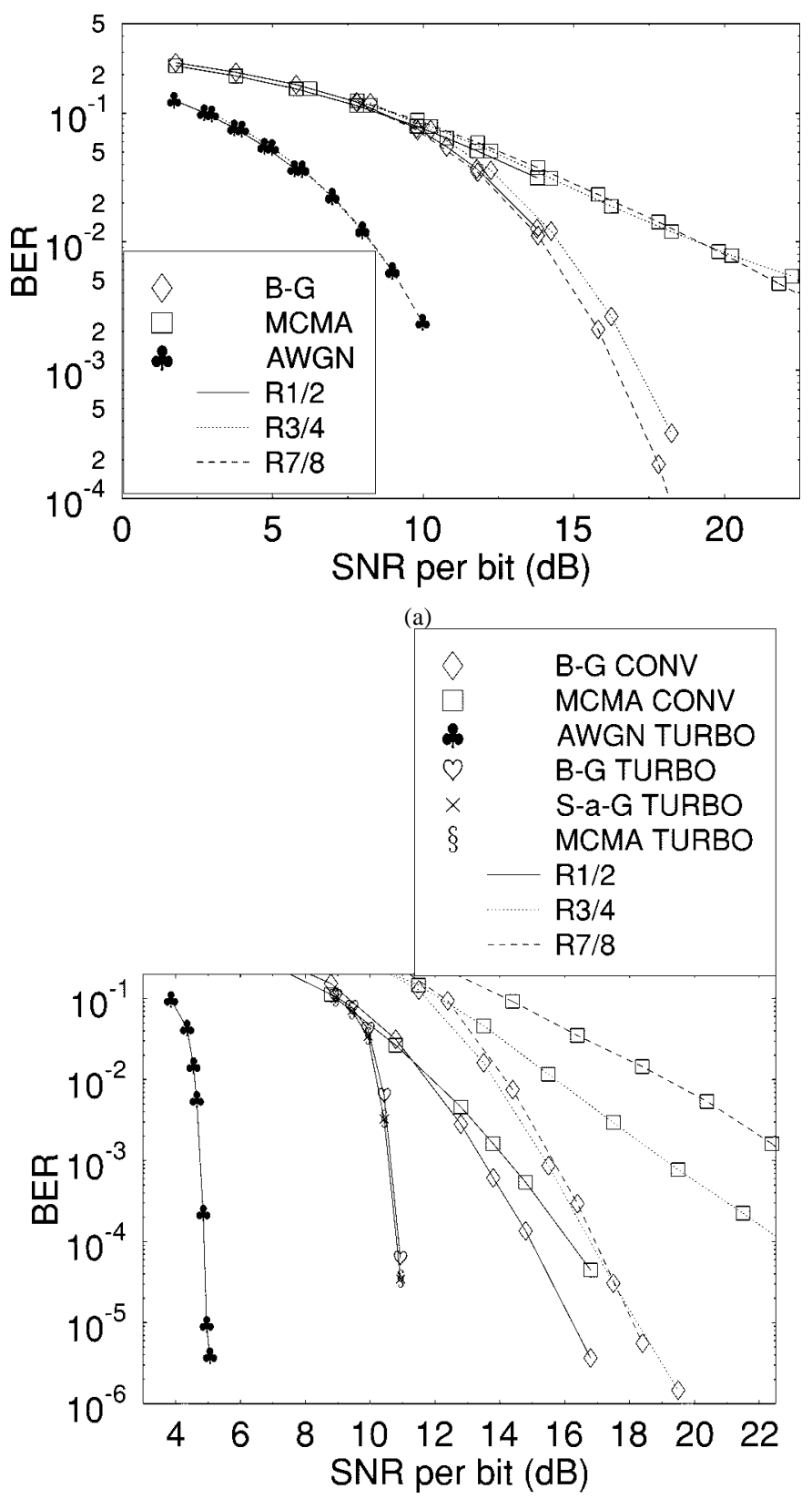

(b)

Fig. 11. Average BER versus SNR per bit performance (a) after equalization and demodulation and (b) after Viterbi or turbo decoding for 16-QAM over the two-symbol delay two-path channel of Fig. 3 (B-G: Benveniste-Goursat; S-a-G: Stop-and-Go; MCMA: Modified Constant Modulus Algorithm; PSP: Per-Survivor-Processing). (a) After equalization and demodulation; (b) After viterbi or turbo decoding.

Again, for simplicity, only the Modified CMA results are given. In this case the ranking order of the different coding rates followed our expectations more closely in the sense that the lowest coding rate of $1 / 2$ was the best performer, followed by rate $3 / 4$ codec, in turn followed by the least powerful rate $7 / 8$ codec.

The Stop-and-Go algorithm has been excluded from these results, since it does not converge for high SNR values. This happens, because the equalization procedure is only activated, when there is a high probability of correct decision-directed equalizer update. In our case, the equalizer is initialized far from its convergence point and hence the decision-directed updates

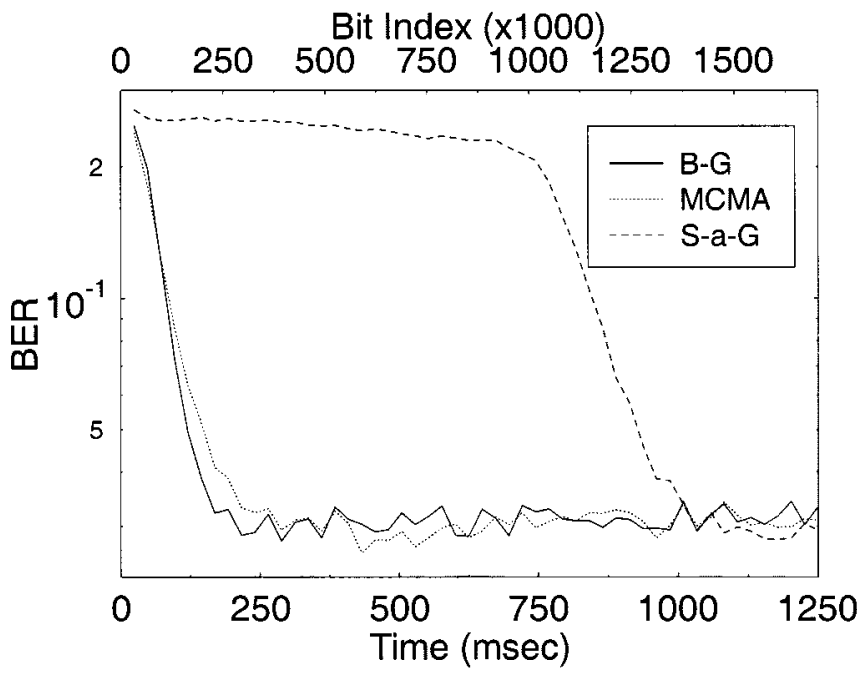

Fig. 12. Learning curves for 16-QAM, one-symbol delay two-path channel at $\mathrm{SNR}=18 \mathrm{~dB}$.

are unlikely to be correct. In the absence of noise this leads to the update algorithm being permanently de-activated. If noise is present though, then some random perturbations from the point of the equalizers initialization can activate the Stop-and-Go algorithm and can lead to convergence. We made this observation at medium SNR values in our simulation study. For high SNR values though, the algorithm did not converge.

It is also interesting to compare the performance of the system for the QPSK and 16-QAM schemes. When the one-symbol delay two-path channel model of Fig. 3 was considered, the system was capable of supporting the use of 16-QAM with the provision of an additional SNR per bit of approximately 4-5 $\mathrm{dB}$. This observation was made by comparing the performance of the DVB system when employing the Modified CMA and the half-rate convolutional or turbo code in Figs. 6 and 8 at a BER of $10^{-4}$. Although the original DVB-Satellite system only employs QPSK modulation, our simulations had shown that 16-QAM can be employed equally well for the range of blind equalizers that we have used in our work. This allowed us to double the video bitrate and hence to substantially improve the video quality. The comparison of Figs. 6 and 8 also reveals the extra SNR requirement of approximately $4-5 \mathrm{~dB}$ of 16QAM over QPSK can be eliminated by employing turbo coding at the cost of a higher implementational complexity. This allowed us to accommodate a doubled bitrate within a given bandwidth, which improved the video quality.

\section{B. Transmission Over the Two-Symbol Delay Two-Path Channel}

In Figs. 9 (only for the Benveniste-Goursat algorithm for simplicity) and 10 the corresponding BER results for the twosymbol delay two-path channel of Fig. 3 are given for QPSK. The associated trends are similar to those in Figs. 4 and 6, although some differences can be observed, as listed below:

- The "cross-over point," beyond which the performance of the PSP algorithm was inferior to that of the Modified CMA in conjunction with turbo decoding is now at $10^{-4}$, 
which is in the range, where the RS decoder guarantees an extremely low probability of error.

- The rate $1 / 2$ convolutional decoding was now the best performer, when convolutional decoding is concerned, while the rate 3/4 scheme exhibited the worst performance.

Finally, in Fig. 11, the associated 16-QAM results are presented. Notice that the Stop-and-Go algorithm was again excluded from the results. Furthermore, we observe a high performance difference between the Benveniste-Goursat algorithm and the Modified CMA. In the previous cases we did not observe such a significant difference. The difference in this case is that the channel exhibits an increased delay spread. This illustrated the capability of the equalizers to cope with more widespread multipaths, while keeping the equalizer order constant at 10. The Benveniste-Goursat equalizer was more efficient, than the Modified CMA in this case.

It is interesting to note that in this case, the performance of the different coding rates was again in the expected order, the rate $1 / 2$ being the best, followed by the rate $3 / 4$ and then the rate $7 / 8$ scheme.

If we compare the performance of the system employing QPSK and 16-QAM over the two-symbol delay two-path channel of Fig. 3, we again observe that 16-QAM can be incorporated into the DVB system, if an extra $5 \mathrm{~dB}$ of SNR per bit is affordable in power budget terms. However, only the $\mathrm{B}-\mathrm{G}$ algorithm is worthwhile considering here out of the three linear equalizers of Table III.This observation was made by comparing the performance of the DVB system when employing the Benveniste-Goursat equalizer and the half-rate convolutional coder in Figs. 10 and 11.

\section{Performance Summary of the DVB-S System}

Table IV provides an approximation of the convergence speed of each blind equalization algorithm of Table III. It is clear that PSP exhibited the fastest convergence, followed by the Benveniste-Goursat algorithm. In our simulations the convergence was quantified by observing the slope of the BER curve, and finding when this curve was reaching the associated residual BER, implying that the BER has reached its steady-state value. Fig. 12 gives an illustrative example of the equalizers convergence for 16-QAM. The Stop-and-Go algorithm converges significantly slower than the other algorithms, which can also be seen from Table IV. This happens because, during the startup, the algorithm is de-activated most of the time, an effect which becomes more severe with an increasing QAM order.

Fig. 13 portrays the corresponding reconstructed video quality in terms of the average peak signal-to-noise ratio (PSNR) versus channel SNR (CSNR) for the one-symbol delay and two-symbol delay two-path channel model of Fig. 3. The PSNR is defined as follows:

$$
\text { PSNR }=10 \log _{10} \frac{\sum_{n=0}^{N} \sum_{m=0}^{M} 255^{2}}{\sum_{n=0}^{N} \sum_{m=0}^{M} \Delta^{2}},
$$

TABLE IV

EQualizer Convergence SPEed (IN Milliseconds) MEasured IN THE SiMUlations, Given AS AN ESTIMATE of TIME REQUIRED For CONVERGENCE WHEN 1/2 RATE PUNCTURING IS USED (x sym: $x$-SyMbOL DELAY TWO-PATH Channel and x Can TaKe EITHer the Value 1 OR 2)

\begin{tabular}{|l|l|l|l|l|}
\hline & B-G & MCMA & S-a-G & PSP \\
\hline QPSK 1 sym & 73 & 161 & 143 & 0.139 \\
\hline QPSK 2 sym & 73 & 143 & 77 & 0.139 \\
\hline 16-QAM 1 sym & 411 & 645 & 1393 & \\
\hline 16-QAM 2 sym & 359 & 411 & 1320 & \\
\hline
\end{tabular}

TABLE V

Summary of Performance Results OVER THE DisPersive One-Symbol DELAY TWO-PATH AWGN ChaNNEL OF FIG. 3 TOLERATING A PSNR DEGRADATION OF $2 \mathrm{~dB}$

\begin{tabular}{|l|l|l|r|r|}
\hline Mod. & Equaliser & Code & $\begin{array}{r}\text { CSNR } \\
(\mathrm{dB})\end{array}$ & $E_{b} / N_{0}$ \\
\hline \hline QPSK & PSP & R=1/2 & 5.3 & 5.3 \\
\hline QPSK & MCMA & Turbo $(1 / 2)$ & 5.2 & 5.2 \\
\hline 16QAM & MCMA & Turbo $(1 / 2)$ & 13.6 & 10.6 \\
\hline QPSK & MCMA & Conv $(1 / 2)$ & 9.1 & 9.1 \\
\hline 16QAM & MCMA & Conv $(1 / 2)$ & 17.2 & 14.2 \\
\hline QPSK & MCMA & Conv $(3 / 4)$ & 11.5 & 9.7 \\
\hline 16QAM & MCMA & Conv $(3 / 4)$ & 20.2 & 15.4 \\
\hline QPSK & B-G & Conv $(7 / 8)$ & 13.2 & 10.8 \\
\hline 16QAM & B-G & Conv $(7 / 8)$ & 21.6 & 16.2 \\
\hline
\end{tabular}

TABLE VI

SUMmary OF PERFORMANCE RESULTS OVER THE DISPERSIVE TwO-SYMBOL DELAY TWO-PATH AWGN ChANNEL OF FIG. 3 TOLERATING A PSNR DEGRADATION OF $2 \mathrm{~dB}$

\begin{tabular}{|l|l|l|r|r|}
\hline Mod. & Equaliser & Code & $\begin{array}{r}\text { CSNR } \\
(\mathrm{dB})\end{array}$ & $E_{b} / N_{0}$ \\
\hline \hline QPSK & PSP & $\mathrm{R}=1 / 2$ & 4.7 & 4.7 \\
\hline QPSK & B-G & Turbo $(1 / 2)$ & 5.9 & 5.9 \\
\hline 16QAM & B-G & Turbo (1/2) & 13.7 & 10.7 \\
\hline QPSK & B-G & Conv $(1 / 2)$ & 8.0 & 8.0 \\
\hline 16QAM & B-G & Conv $(1 / 2)$ & 17.0 & 14.0 \\
\hline QPSK & B-G & Conv $(3 / 4)$ & 12.1 & 10.3 \\
\hline 16QAM & B-G & Conv $(3 / 4)$ & 21.1 & 16.3 \\
\hline QPSK & B-G & Conv $(7 / 8)$ & 13.4 & 11.0 \\
\hline 16QAM & MCMA & Conv $(7 / 8)$ & 29.2 & 23.8 \\
\hline
\end{tabular}

where $\Delta$ is the difference between the uncoded pixel value and the reconstructed pixel value. The variables $M$ and $N$ refer to the dimension of the image. The maximum possible 8-bit represented pixel luminance value of 255 was used in (19) in order to mitigate the PSNR's dependence on the video material used. The average PSNR is then the mean of the PSNR values computed for all the images constituting the video sequence.

Tables V and VI provide a summary of the DVB-Satellite system's performance tolerating a PSNR degradation of $2 \mathrm{~dB}$, which was deemed to be nearly imperceptible in terms of subjective video degradations. The average BER values quoted in the tables refer to the average BER achieved after Viterbi or turbo decoding. The channel SNR is quoted in association with the $2 \mathrm{~dB}$ average video PSNR degradation, since the viewer will begin to perceive video degradations due to erroneous decoding of the received video around this threshold. 


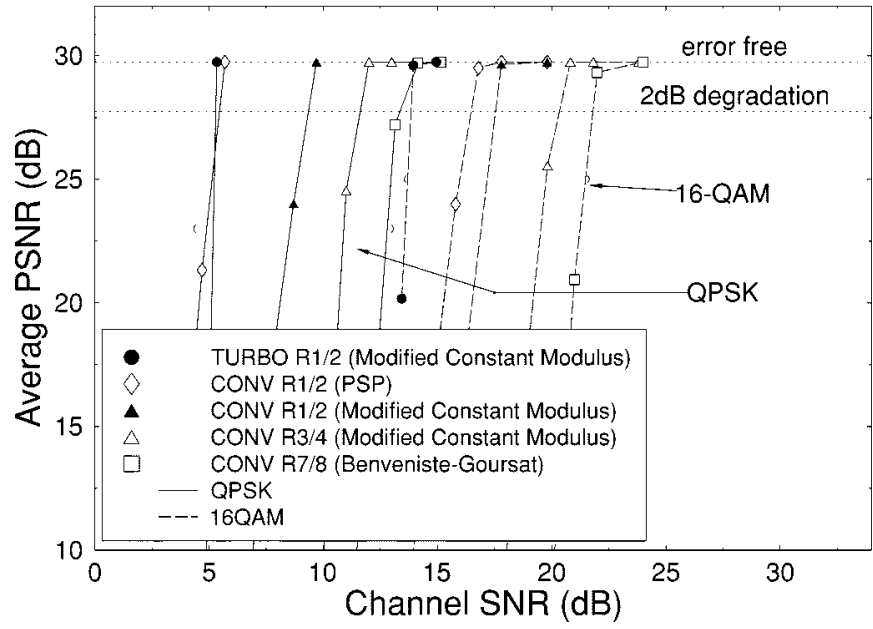

(a)

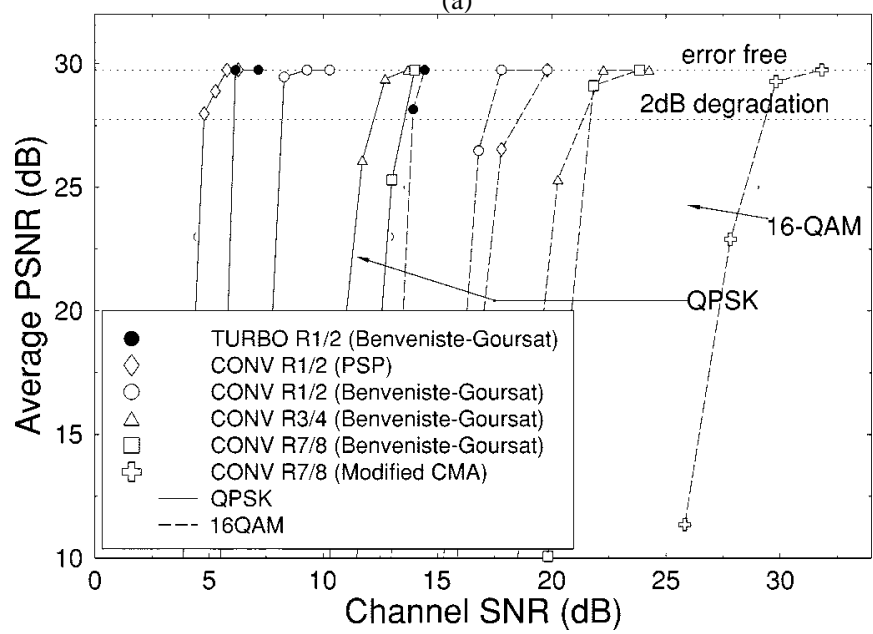

(b)

Fig. 13. Average PSNR versus channel SNR for: (a) the one-symbol delay two-path channel model, and (b) the two-symbol delay two-path channel model of Fig. 3 at a video bitrate of $2.5 \mathrm{Mbit} / \mathrm{s}$ using the "Football" sequence. (a) One-symbol delay two-path channel model; (b) Two-symbol delay two-path channel model.

Tables VII and VIII provide a summary of the SNR per bit required for the various system configurations. The BER threshold of $10^{-4}$ was selected here, since at this average BER after Viterbi or turbo decoding, the RS decoder becomes effective, guaranteeing near error-free performance. This also translates into near unimpaired reconstructed video quality.

Finally, in Table IX the QAM symbol rate or Baud rate is given for different puncturing rates and for different modulation schemes, based on the requirement of supporting a video bit rate of $2.5 \mathrm{Mbit} / \mathrm{sec}$. We observe that the Baud rate is between 0.779 and $2.73 \mathrm{MBd}$, depending on the coding rate and the number of bits per modulation symbol.

\section{CONClUSIONS AND FUtURE WORK}

In this contribution, we have investigated the performance of a turbo-coded DVB system in a satellite broadcast environment. A range of system performance results was presented based on the standard DVB-S scheme, as well as on a turbo-coded scheme in conjunction with blind-equalized QPSK/16QAM. The convolutional code specified in the standard system was substituted
TABLE VII

Summary of System Performance Results Over the Dispersive ONE-SyMbol DELAY TWo-PATH AWGN CHANNEl OF Fig. 3 TOLERATING AN AVERAGE BER OF $10^{-4}$, WHICH WAS EVALUATED AFTER VITERBI OR TuRbo DeCoding BUt Before RS DECODING

\begin{tabular}{|l|l|l|r|}
\hline Mod. & Equaliser & Code & $E_{b} / N_{0}$ \\
\hline \hline QPSK & PSP & R=1/2 & 6.1 \\
\hline QPSK & MCMA & Turbo (1/2) & 5.2 \\
\hline 16QAM & MCMA & Turbo (1/2) & 10.7 \\
\hline QPSK & MCMA & Conv (1/2) & 11.6 \\
\hline 16QAM & MCMA & Conv (1/2) & 15.3 \\
\hline QPSK & MCMA & Conv (3/4) & 10.5 \\
\hline 16QAM & MCMA & Conv (3/4) & 16.4 \\
\hline QPSK & B-G & Conv $(7 / 8)$ & 11.8 \\
\hline 16QAM & B-G & Conv $(7 / 8)$ & 17.2 \\
\hline
\end{tabular}

TABLE VIII

SuMmary of System PERfoRMANCE RESUlTS OVER THE DISPERSIVE ONE-Symbol Delay Two-Path AWGN ChanNEl OF Fig. 3 TOLERATING aN AVERAGE BER OF $10^{-4}$, WHICH WAS EVALUATED AFTER VITERBI OR TuRbo DECODING BUt BEFore RS DECODING

\begin{tabular}{|l|l|l|r|}
\hline Mod. & Equaliser & Code & $E_{b} / N_{0}$ \\
\hline \hline QPSK & PSP & R=1/2 & 5.6 \\
\hline QPSK & B-G & Turbo (1/2) & 5.7 \\
\hline 16QAM & B-G & Turbo (1/2) & 10.7 \\
\hline QPSK & B-G & Conv (1/2) & 9.2 \\
\hline 16QAM & B-G & Conv (1/2) & 15.0 \\
\hline QPSK & B-G & Conv (3/4) & 12.0 \\
\hline 16QAM & B-G & Conv (3/4) & 16.8 \\
\hline QPSK & B-G & Conv (7/8) & 11.7 \\
\hline 16QAM & MCMA & Conv $(7 / 8)$ & 26.0 \\
\hline
\end{tabular}

TABLE IX

The Channel Bit Rate For the Three DifFerent Punctured Coding RATES AND FOR THE TWO MODULATIONS

\begin{tabular}{|c|c|c|}
\hline Punctured Rate & $\begin{array}{c}\text { 4-QAM } \\
\text { Baud Rate } \\
\text { (MBd) }\end{array}$ & $\begin{array}{c}\text { 16-QAM } \\
\text { Baud Rate } \\
\text { (MBd) }\end{array}$ \\
\hline $1 / 2$ & 2.73 & 1.37 \\
\hline $3 / 4$ & 1.82 & 0.909 \\
\hline $7 / 8$ & 1.56 & 0.779 \\
\hline
\end{tabular}

with turbo coding, which resulted in a substantial coding gain of approximately 4-5 $\mathrm{dB}$. We have also shown that 16-QAM can be utilized instead of QPSK, if an extra $5 \mathrm{~dB}$ SNR per bit gain is added to the link budget. This extra transmitted power requirement can be eliminated upon invoking the more complex turbo codec, which requires lower transmitted power for attaining the same performance as the standard convolutional codecs.

Our future work will be focused on extending the DVB-Satellite system to supporting mobile users for the reception of satellite broadcast signals. The use of blind turbo equalizers will also be investigated in comparison to conventional blind equalizers. Further work will also be dedicated to trellis coded modulation (TCM) and turbo trellis coded modulation (TTCM) based orthogonal frequency division multiplexed (OFDM) and singlecarrier equalized modems. The impact on the system performance by employing various types of turbo interleavers and 
turbo coded is also of interest. A range of further wireless video communications issues are addressed in [30].

\section{ACKNOWLEDGMENT}

The authors would like to thank the reviewers for their constructive critique.

\section{REFERENCES}

[1] ETSI, "Digital Video Broadcasting (DVB); Framing structure, channel coding and modulation for digital terrestrial television,", EN 300744 V1.1.2, August 1997

[2] —_, "Digital Video Broadcasting (DVB); Framing structure, channel coding and modulation for cable systems,", EN 300429 V1.2.1, December 1997.

[3] — - "Digital Video Broadcasting (DVB); Framing structure, channel coding and modulation for 11/12 GHz Satellite Services,", EN 300421 V1.1.2, August 1997

[4] R. Steele and L. Hanzo, Eds., Mobile Radio Communications, 2 ed: John Wiley \& Sons/IEEE Press, 1999.

[5] A. M. Michelson and A. H. Levesque, Error Control Techniques for Digital Communication: Wiley-Interscience, 1985.

[6] L. Hanzo, W. T. Webb, and T. Keller, Single and Multicarrier Quadrature Amplitude Modulation: John Wiley \& Sons/IEEE Press, 1999.

[7] G. Reali, G. Baruffa, S. Cacopardi, and F. Frescura, "Enhancing satellite broadcasting services using multiresolution modulations," IEEE Transactions on Broadcasting, vol. 44, pp. 497-506, December 1998.

[8] Y. F. Hsu, Y. C. Chen, C. J. Huang, and M. J. Sun, "MPEG-2 spatial scalable coding and transport stream error concealment for satellite TV broadcasting using Ka-band," IEEE Transactions on Broadcasting, vol. 44, no. 1, pp. 77-86, March 1998.

[9] L. Atzori, F. G. B. De Natale, M. Di Gregario, and D. D. Giusto, "Multimedia information broadcasting using digital TV channels," IEEE Transactions on Broadcasting, vol. 43, no. 4, pp. 383-392, December 1997

[10] W. Sohn, O. H. Kwon, and J. S. Chae, "Digital DBS system design and implementation for TV and data broadcasting using Koreasat," IEEE Transactions on Broadcasting, vol. 44, no. 3, pp. 316-323, September 1998.

[11] C. Berrou, A. Glavieux, and P. Thitimajshima, "Near Shannon limit error-correcting coding and decoding: turbo codes," in IEEE Proceedings of the International Conference on Communications, Geneva, Switzerland, May 1993, pp. 1064-1070.

[12] C. Berrou and A. Glavieux, "Near optimum error correcting coding and decoding: turbo codes," IEEE Transactions on Communications, vol. 44, no. 10, pp. 1261-1271, October 1996.

[13] S. B. Wicker, Error Control Systems for Digital Communication and Storage: Prentice Hall, 1994.

[14] P. Robertson, E. Villebrun, and P. Hoeher, "A comparison of optimal and suboptimal MAP decoding algorithms operating in the log domain," in IEEE Proceedings of the International Conference on Communications, June 1995, pp. 1009-1013.

[15] J. Griffiths, Radio Wave Propagation and Antennas-An Introduction: Prentice Hall, 1987.

[16] M. S. Karaliopoulos and F.-N. Pavlidou, "Modeling the land mobile satellite channel: A review," Electronics and Communication Engineering Journal, vol. 11, no. 5, pp. 235-248, 1999.

[17] J. Goldhirsh and W. J. Vogel, "Mobile satellite system fade statistics for shadowing and multipath from roadside trees at UHF and L-band," IEEE Transactions on Antennas and Propagation, vol. 37, no. 4, pp. 489-498, April 1989.

[18] W. J. Vogel and J. Goldhirsh, "Multipath fading at L band for low elevation angle, land mobile satellite scenarios," IEEE Journal on Selected Areas in Communications, vol. 13, no. 2, pp. 197-204, February 1995.

[19] W. J. Vogel and G. W. Torrence, "Propagation measurements for satellite radio reception inside buildings," IEEE Transactions on Antennas and Propagation, vol. 41, no. 7, pp. 954-961, July 1993.

[20] W. J. Vogel and U. S. Hong, "Measurement and modeling of land mobile satellite propagation at UHF and L-band," IEEE Transactions on Antennas and Propagation, vol. 36, no. 5, pp. 707-719, May 1988.

[21] S. R. Saunders, C. Tzaras, and B. G. Evans, "Physical Statistical Propagation Model for Mobile Satellite Channel," European Commission, Tech. Rep., 1998.
[22] S. Saunders, Antennas and Propagation for Wireless Communication Systems Concept and Design: John Wiley \& Sons, 1999.

[23] K. Wesolowsky, "Analysis and properties of the modified constant modulus algorithm for blind equalization," European Transactions on Telecommunication, vol. 3, no. 3, pp. 225-230, May/June 1992.

[24] M. Goursat and A. Benveniste, "Blind equalizers," IEEE Transactions on Communications, vol. COM-28, no. 11, pp. 871-883, August 1984.

[25] G. Picchi and G. Prati, "Blind equalization and carrier recovery using a "stop-and-go" decision-directed algorithm," IEEE Transactions on Communications, vol. COM-35, no. 9, pp. 877-887, September 1987.

[26] A. Polydoros, R. Raheli, and C. Tzou, "Per-survivor processing: A general approach to MLSE in uncertain environments," IEEE Transactions on Communications, vol. COM-43, no. 2-4, pp. 354-364, February/April 1995.

[27] D. N. Godard, "Self-recovering equalization and carrier tracking in twodimensional data communication systems," IEEE Transactions on Communications, vol. COM-28, no. 11, pp. 1867-1875, November 1980

[28] Y. Sato, "A method of self-recovering equalization for multilevel amplitude-modulation systems," IEEE Transactions on Communications, vol. COM-23, pp. 679-682, June 1975.

[29] Z. Ding, R. A. Kennedy, B. D. O. Anderson, and R. C. Johnson, "Illconvergence of Godard blind equalizers in data communications systems," IEEE Transactions on Communications, vol. COM-39, no. 9, pp. 1313-1327, September 1991.

[30] L. Hanzo, P. J. Cherriman, and J. Streit, Modern Video Compression and Communications: Principles and Applications for Fixed and Wireless Channels: IEEE Press, 1998, to be published.

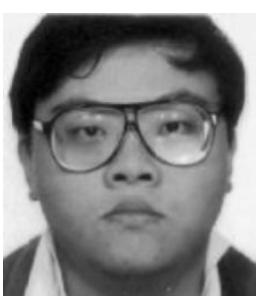

Chee-Siong Lee received a first class honors degree in electrical engineering at the University of Southampton in 1997. Since then, he has been with the Wireless Multimedia Communications Group at the University of Southampton, working toward the $\mathrm{Ph} . \mathrm{D}$. degree in mobile multimedia communications. His current areas of interest include video compression and various aspects of communications.

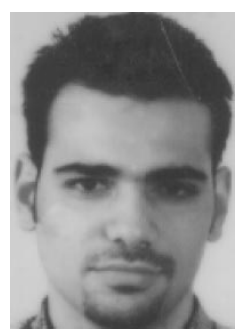

Spyros Vlahoyiannatos studied electrical and computer engineering at the National Technical University of Athens and graduated in 1997. He is currently working toward the Ph.D. degree in the Wireless Multimedia Communications Group at the University of Southampton. His area of research is the blind equalization of mobile fading channels.

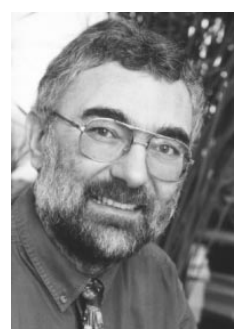

Lajos Hanzo has held various research and academic posts in Hungary, Germany and the UK, during his 24 -year career in telecommunications. Since 1986, he has been a Member of the Academic Staff of the Department of Electronics and Computer Science, University of Southampton, UK and has been a consultant to Multiple Access Communications Ltd., UK. Currently, he holds the Chair in Telecommunications. As a Member of a multinational consortium and funded by the European Community as well as by the Engineering and Physical Sciences Research Council (EPSRC) UK, he is currently conducting research toward the next generation of wireless multimedia systems. He is widely published in Wireless Multimedia Communications, including three monographs and more than 200 research papers, organized and chaired conference sessions, presented overview lectures, and was awarded a number of distinctions. He manages an academic research team. He is a Member of the IEE and Senior Member of the IEEE. For further information please refer to http://www-mobile.ecs.soton.ac.uk 